\title{
LOCAL CUT-POINTS IN CONTINUOUS IMAGES OF COMPACT ORDERED SPACES
}

\author{
RYUJI MAEHARA
}

\begin{abstract}
We prove that if a continuum $X$ is continuous image of a compact ordered space and if $X$ is not locally separable at a point $x$, then $x$ lies in the closure of the set of all local cut-points of $X$.
\end{abstract}

A continuum is a compact connected Hausdorff space. An ordered space is a totally ordered set endowed with the topology generated by open intervals. It is known from L. B. Treybig [4] that if a nonmetrizable continuum $X$ is a continuous image of a compact ordered space, then some subset of less than three points separates $X$. In this paper we prove the following theorem by modifying Treybig's method, and give a corollary.

THEOREM. If a continuum $X$ is a continuous image of a compact ordered space and if $X$ is not locally separable at $x_{0}$, then for any neighborhood $U$ of $x_{0}$ there exists a nonempty open set $W$ such that $\bar{W} \subset U$ and the boundary $\operatorname{Bd}(W)$ consists of at most two points.

A space $X$ is said to be locally separable at $x$ if there is a separable neighborhood of $x$. A point $x$ of $X$ is a local cut-point of $X$ if there is a connected open neighborhood $U$ of $x$ such that $U-\{x\}$ is not connected. We will denote the set of all local cut-points of $X$ by $L(X)$. If $W$ is a nonempty open subset of a continuum $X$ such that its closure $\bar{W}$ is not $X$, then its boundary $\operatorname{Bd}(W)$ separates $X$. In addition, if $\operatorname{Bd}(W)$ is finite, $\operatorname{Bd}(W)$ contains at least one local cut-point. Hence, the theorem implies:

COROllary. Let $X$ be a continuum which is a continuous image of a compact ordered space. If $X$ is not locally separable at $x_{0}$, then $x_{0}$ is in the closure of $L(X)$.

In the preceding corollary, even if $X$ is locally connected, we can assert neither that $x_{0}$ is always an accumulation point of $L(X)$, nor that $x_{0}$ is a local cut-point or an end-point of $X$. Counterexamples for these will be given in $\S 3$.

1. We prepare an essential part of Treybig's method used in [4 and 5] in a slightly modified form as follows:

LEMMA. Let $K$ be a nonseparable compact ordered space, and let $f$ be a continuous map from $K$ onto a Hausdorff space. Given a countable subset $L_{0}$ of $K, K$ can be

Received by the editors October 28, 1985 and, in revised form, February 10, 1986.

1980 Mathematics Subject Classification (1985 Revision). Primary 54F25; Secondary 54F05.

Key words and phrases. Compact ordered space, local cut-point, local separability. 
written as the union of mutually disjoint nonempty subsets,

$$
K=L \cup\left(\bigcup_{\lambda} G_{\lambda}\right)
$$

such that the following conditions are satisfied:

(1) $L \cap G_{\lambda}=\varnothing, G_{\lambda} \cap G_{\mu}=\varnothing($ if $\lambda \neq \mu)$,

(2) $L$ is a separable closed set containing $L_{0}$,

(3) each $G_{\lambda}$ is an open set of the form $\left(a_{\lambda}, b_{\lambda}\right)$,

(4) if $f\left(G_{\lambda}\right) \cap f\left(G_{\mu}\right) \neq \varnothing$, then $\left\{f\left(a_{\lambda}\right), f\left(b_{\lambda}\right)\right\}=\left\{f\left(a_{\mu}\right), f\left(b_{\lambda}\right)\right\}$, and

(5) $f(L) \cap f\left(G_{\lambda}\right) \subset\left\{f\left(a_{\lambda}\right), f\left(b_{\lambda}\right)\right\}$.

Proof. Let $L_{0}=\left\{c_{1}, c_{2}, c_{3}, \ldots\right\}$. We construct a sequence of finite subsets $L_{1}$, $L_{2}, L_{3}, \ldots$ of $K$ inductively as follows. Let $a_{0}, a_{2}$ be the minimum and the maximum of $K$, respectively. Choose $a_{1}$ so that $a_{0}<a_{1}<a_{2}$, and define $L_{1}=$ $\left\{a_{0}, a_{1}, a_{2}\right\}$. Suppose that $L_{n}=\left\{b_{0}, b_{1}, \ldots, b_{p}\right\}$ is defined, where $b_{0}<b_{1}<\cdots$ $<b_{p}$. Put $I_{i}=\left[b_{i-1}, b_{i}\right]$ for $i=1,2, \ldots, p$. If $i \neq j$, we define

$$
A_{i j}=\left\{(\xi, \eta) \in I_{i} \times I_{j}: f(\xi)=f(\eta)\right\} .
$$

If $A_{i j}$ is nonempty, there exists a subset $\left\{\left(\alpha, \alpha^{\prime}\right),\left(\beta, \beta^{\prime}\right),\left(\gamma^{\prime}, \gamma\right),\left(\delta^{\prime}, \delta\right)\right\}$ of $A_{i j}$ such that

$$
\alpha \leqslant \xi \leqslant \beta, \quad \gamma \leqslant \eta \leqslant \delta \quad \text { for all }(\xi, \eta) \in A_{i j} .
$$

We set $B_{i j}=\left\{\alpha, \alpha^{\prime}, \beta, \beta^{\prime}, \gamma^{\prime}, \gamma, \delta^{\prime}, \delta\right\}$. If $A_{i j}$ is empty we set $B_{i j}=\varnothing$. Define

$$
L_{n+1}=L_{n} \cup\left(\bigcup_{i \neq j} B_{i j}\right) \cup\left\{c_{1}, c_{2}, \ldots, c_{n}\right\},
$$

and let $L=\bar{U}_{n} L_{n}$. Then $L$ is a separable closed set. Since $K$ is nonseparable, $K-L$ is a nonempty open subset of $K . K-L$ can be written as the union of mutually disjoint maximal convex open subsets $G_{\lambda}$. Since the minimum and the maximum of $K$ belong to $L$, each $G_{\lambda}$ is of the form $\left(a_{\lambda}, b_{\lambda}\right)$, where $a_{\lambda}, b_{\lambda} \in L$.

To prove (4), suppose that $f(c)=f(d)$ for $c \in G_{\lambda}$ and $d \in G_{\mu}$. Also suppose that $f\left(a_{\lambda}\right) \notin\left\{f\left(a_{\mu}\right), f\left(b_{\mu}\right)\right\}$. Then there exist convex open neighborhoods $U_{\lambda}, U_{\mu}, V_{\mu}$ of $a_{\lambda}, a_{\mu}, b_{\mu}$, respectively, such that

$$
f\left(U_{\lambda}\right) \cap f\left(U_{\mu}\right)=\varnothing \text { and } f\left(U_{\lambda}\right) \cap f\left(V_{\mu}\right)=\varnothing .
$$

By the definition of $L$, for some integer $n$ the set $L_{n}=\left\{b_{0}, b_{1}, \ldots, b_{p}\right\}$ contains elements $b_{i}, b_{j}$ such that

$$
\begin{gathered}
\left(a_{\lambda}, b_{\lambda}\right) \subset\left[b_{i-1}, b_{i}\right], \quad\left(a_{\mu}, b_{\mu}\right) \subset\left[b_{j-1}, b_{j}\right], \\
b_{i-1} \in U_{\lambda}, \quad b_{j-1} \in U_{\mu}, \quad b_{j} \in V_{\mu} .
\end{gathered}
$$

Since $f(c)=f(d)$, by construction, the set $L_{n+1}$ contains elements $\alpha, \alpha^{\prime} \in B_{i j}$ such that

$$
b_{i-1} \leqslant \alpha \leqslant c, \quad b_{j-1} \leqslant \alpha^{\prime} \leqslant b_{j}, \quad \text { and } \quad f(\alpha)=f\left(\alpha^{\prime}\right) .
$$

If $a_{\lambda}<\alpha$, then we would have $a_{\lambda}<\alpha<b_{\lambda}$ and hence $\alpha \in G_{\lambda} \cap L_{n+1}$ (contradiction). If $\alpha \leqslant a_{\lambda}$, then $\alpha \in U_{\lambda}$. So $\alpha^{\prime}$ could not lie in $U_{\mu} \cup V_{\mu}$, and hence $a_{\mu}<\alpha^{\prime}<b_{\mu}$ (contradiction). A similar argument shows that $f\left(b_{\lambda}\right) \in\left\{f\left(a_{\mu}\right), f\left(b_{\mu}\right)\right\}$ and $\left\{f\left(a_{\mu}\right), f\left(b_{\mu}\right)\right\} \subset\left\{f\left(a_{\lambda}\right), f\left(b_{\lambda}\right)\right\}$. 
To prove (5), suppose that $f(c)=f(d)$ for $c \in L$ and $d \in G_{\lambda}$, and that $f(c) \notin$ $\left\{f\left(a_{\lambda}\right), f\left(b_{\lambda}\right)\right\}$. Choose convex neighborhoods $W, U_{\lambda}, V_{\lambda}$ of $c, a_{\lambda}, b_{\lambda}$, respectively so that

$$
f(W) \cap f\left(U_{\lambda}\right)=\varnothing, \quad f(W) \cap f\left(V_{\lambda}\right)=\varnothing .
$$

For some integer $n$, the set $L_{n}=\left\{b_{0}, b_{1}, \ldots, b_{p}\right\}$ contains such elements $b_{i}, b_{j}$ that satisfy:

$$
\begin{gathered}
b_{i-1} \leqslant c \leqslant b_{i}, \quad b_{j-1} \leqslant a_{\lambda}<d<b_{\lambda} \leqslant b_{j}, \\
b_{j-1} \in U_{\lambda}, \quad b_{j} \in V_{\lambda}, \quad \text { and } \quad\left\{b_{i-1}, b_{i}\right\} \cap W \neq \varnothing .
\end{gathered}
$$

Then the set $L_{n+1}$ contains elements $\alpha, \alpha^{\prime}, \beta, \beta^{\prime}$ such that

$$
\begin{gathered}
b_{i-1} \leqslant \alpha \leqslant c \leqslant \beta \leqslant b_{i}, \quad \alpha^{\prime}, \beta^{\prime} \in\left[b_{j-1}, b_{j}\right], \\
f(\alpha)=f\left(\alpha^{\prime}\right) \text { and } f(\beta)=f\left(\beta^{\prime}\right) .
\end{gathered}
$$

If $b_{i-1} \in W$, then $\alpha \in W$ and so $\alpha^{\prime} \notin U_{\lambda} \cup V_{\lambda}$, whence $\alpha^{\prime} \in\left(a_{\lambda}, b_{\lambda}\right)$. This is a contradiction. Similarly, if $b_{i} \in W$, then we would have $\beta^{\prime} \in\left(a_{\lambda}, b_{\lambda}\right)$.

2. Proof of the theorem. We assume $U \neq X$. Since $X$ is normal, we can find a sequence of open sets $U_{1}, U_{2}, U_{3}, \ldots$ such that

$$
x_{0} \in U_{n} \subset \bar{U}_{n} \subset U_{n+1} \subset \bar{U}_{n+1} \subset U \text {. }
$$

Let $S=X-\left(\cup\left\{U_{n}: n=1,2,3, \ldots\right\}\right)$; then $S$ is a nonempty closed set and $x_{0} \notin S$. We define an equivalence relation $\sim$ on $X$ by setting $x \sim y$ if and only if $x=y$ or $\{x, y\} \subset S$. Since $\sim$ is a closed relation, the quotient space $X_{1}=X / \sim$ is Hausdorff. Note that the natural projection $\phi: X \rightarrow X_{1}$ is a local homeomorphism on $X-S$, and hence, $X_{1}$ is not separable. Let $V_{n}=\phi\left(X-\bar{U}_{n}\right)$. Then $\left\{V_{n}\right\}_{n=1}^{\infty}$ forms a countable basis of neighborhoods at point $s(=\phi(S))$ in $X_{1}$.

As well as $X, X_{1}$ is a continuous image of compact ordered space. Let $K$ be a compact ordered space, and $f$ a continuous surjection from $K$ onto $X_{1}$. By [3, Lemma 4] we can assume that (i) if $K_{1}$ is a closed proper subset of $K$, then $f\left(K_{1}\right) \neq X_{1}$, and (ii) if $a, b$ are different elements of $K$ with $f(a)=f(b)$, then there is a point $c$ between $a$ and $b$ such that $f(c) \neq f(a)$.

For each positive integer $n$, there is a finite cover $\Omega_{n}$ of $f^{-1}(s)$ such that each member of $\Omega_{n}$ is of the form $\left(a_{i}, b_{i}\right)$, or $\left(a_{i}, \infty\right)$, or $\left(-\infty, b_{i}\right)$, and is contained in $f^{-1}\left(V_{n}\right)$. Let $L_{n}$ denote the set of all end-points $a_{i}, b_{i}$ of members of $\Omega_{n}$, and let $L_{0}=\bigcup_{n} L_{n}$. We remark that if two points $a, b$ of $K$ are not consecutive and $f(a)=s$, then there is a point of $L_{0}$ between $a$ and $b$. In fact, choose a point $c$ between $a$ and $b$. By (ii), we can assume $f(c) \neq s$. There is an integer $n$ such that $f(c) \notin V_{n}$. Then one of the end-points of a member of $\Omega_{n}$ which contains $a$ must lie between $a$ and $b$.

If necessary, adding a point of $f^{-1}(s)$ to $L_{0}$, we can assume $s \in f\left(L_{0}\right)$. Noting that $K$ is nonseparable, we apply our lemma for the map $f: K \rightarrow X_{1}$ and the countable subset $L_{0}$ of $K$ to obtain a decomposition of $K$ into mutually disjoint nonempty subsets with properties (1)-(5) as in the lemma:

$$
K=L \cup\left(\bigcup_{\lambda} G_{\lambda}\right) \text {. }
$$


We choose one $G_{\lambda}$ and denote it by $G=(a, b)$. Let $H^{*}$ be the collection of all $G_{\mu}$ such that $\left\{f\left(a_{\mu}\right), f\left(b_{\mu}\right)\right\}=\{f(a), f(b)\}$, and let $H=\bigcup H^{*}$ and $M=\left\{\mu: G_{\mu} \in\right.$ $\left.H^{*}\right\}$.

Let $W_{1}=X_{1}-f(K-H) . W_{1}$ is open. $W_{1}$ is nonempty, since otherwise we would have $X_{1}=f(K-H)$ where $K-H$ is a proper closed subset of $K$, contradicting assumption (i) on map $f$. Since $W_{1} \subset f(H) \subset f(\bar{H})$, it follows that $\bar{W}_{1} \subset f(\bar{H})$. Now let $c$ be a boundary point of the open set $H$, and let $V$ be an arbitrary convex neighborhood of $c$. Since $V$ meets $H, V$ meets some member $G_{\mu}$ of $H^{*}$. But $c$ is not in $G_{\mu}$. Then one of $a_{\mu}$ or $b_{\mu}$ must lie in $V$. Thus $c$ is in the closure of $\left\{a_{\mu}, b_{\mu}: \mu \in M\right\}$ and hence in the closed set $f^{-1} f(a) \cup f^{-1} f(b)$. Therefore,

$$
\bar{H} \subset H \cup f^{-1} f(a) \cup f^{-1} f(b),
$$

and

$$
\bar{W}_{1} \subset f(\bar{H}) \subset f(H) \cup\{f(a), f(b)\}
$$

It follows that

$$
\begin{aligned}
\operatorname{Bd}\left(W_{1}\right) & =\bar{W}_{1} \cap \overline{X_{1}-W_{1}} \subset(f(H) \cup\{f(a), f(b)\}) \cap f(K-H) \\
& =\{f(a), f(b)\} \quad \text { (by properties (4) and (5)). }
\end{aligned}
$$

Note that $s \notin\{f(a), f(b)\}$, since if for example $f(a)=s$, then as remarked previously, some element of $L_{0}$ would lie in $G=(a, b)$, contradicting $G \cap L=\varnothing$. Thus $s \notin \bar{W}_{1}$. Finally, since $\phi: X \rightarrow X_{1}$ is locally homeomorphic on $X-S$, and $s=\phi(S) \notin \bar{W}_{1}$, the open set $W=\phi^{-1}\left(W_{1}\right)$ of $X$ satisfies the required properties in the theorem.

3. Examples. As mentioned in the introduction, we give two examples to complement our corollary.

EXAMPLE 1. Let $\Lambda$ be an uncountable set. For each $\lambda \in \Lambda$, let $D_{\lambda}$ denote the unit closed disk with center $o$ in the complex number plane, and let $Y$ denote the product of all $D_{\lambda}$. We regard each $D_{\lambda}$ as a subset of $Y$ by identifying a point $z \in D_{\lambda}$ with the point $\left(z_{\mu}\right)$ of $Y$ defined by $z_{\mu}=z$ if $\mu=\lambda$, and $z_{\mu}=o$ if $\mu \neq \lambda$. Then all subsets $D_{\lambda}$ have the common center $O$. By the definition of the product topology, we note that every neighborhood of $O$ contains all $D_{\lambda}$ except for finitely many $\lambda$. Define $X=\cup_{\lambda} D_{\lambda}$ and endow $X$ with the relative topology from $Y$. Then space $X$ has the following properties:

(1) $X$ is a locally connected continuum,

(2) $X$ is not locally separable at $O$, and is locally separable at any other points,

(3) $O$ is a cut-point of $X$ and it is a unique local cut-point,

(4) $X$ is two dimensional at every point, and

(5) $X$ is continuous image of an ordered continuum.

Properties (1)-(4) are easily verified. Property (5) follows from a theorem of Cornette [1] that states that a locally connected continuum is a continuous image of an ordered continuum if and only if its every cyclic element is so. Note that the cyclic elements of $X$ are the $D_{\lambda}$ which are locally connected metric continuum and hence are continuous images of the real interval $[0,1]$ by the classical theorem of Hahn-Mazurkiewicz. 
EXAmpLe 2. Let $I$ denote the interval $[0,1]$ of the real numbers, and $L$ the "long interval" $[0, \Omega]$, which is a nonseparable ordered continuum. We define a subspace $Y$ of $L \times I$ by

$$
Y=A_{0} \cup\left(\bigcup_{n} A_{n}\right) \cup B_{0} \cup B_{\Omega}
$$

where

$$
\begin{aligned}
& A_{0}=\{(\lambda, 0): \lambda \in L\}, \\
& A_{n}=\{(\lambda, 1 / n): \lambda \in L\}, \quad \text { where } n=1,2,3, \ldots, \\
& B_{0}=\{(0, t): t \in I\}, \quad B_{\Omega}=\{(\Omega, t): t \in I\} .
\end{aligned}
$$

Define an equivalence relation $\sim$ on $Y$ by setting $y \sim z$ if and only if $y=z$ or $\{y, z\} \subset A_{0}$. Let $X$ denote the quotient space $Y / \sim$, and $a_{0}$ the point which is the image of $A_{0}$ under the natural projection. Then $X$ satisfies the following properties:

(1) $X$ is a continuum,

(2) $X$ is netlike (that means, any two different points of $X$ can be separated by a finite subset of $X$ ),

(3) $X$ is not locally separable at $a_{0}$,

(4) $a_{0}$ is neither a local cut-point nor an end-point of $X$, and

(5) $X$ is a continuous image of an ordered continuum.

Properties (1)-(4) are easily verified. Property (5) follows from (1) and (2) (see [2 or 6]).

\section{REFERENCES}

1. J. L. Cornete, "Image of a Hausdorff arc" is cyclically extensible and reducible, Trans. Amer. Math. Soc. 199 (1974), 253-267.

2. B. J. Pearson, Mapping arcs and dendritic spaces onto netlike continua, Colloq. Math. 34 (1975), $39-48$.

3. L. B. Treybig, Concerning continuous images of compact ordered spaces, Proc. Amer. Math. Soc. 15 (1964), 866-871.

4. Concerning continua which are continuous images of compact ordered spaces, Duke Math. J. 32 (1965), 417-422.

5. S Separation by finite sets in connected, continuous images of ordered compacta, Proc. Amer. Math. Soc. 74 (1979), 326-328.

6. L. E. Ward, Jr., The Hahn-Mazurkiewicz theorem for rim-finite continua, General Topology Appl. 6 (1976), 183-190.

7. G. T. Whyburn, Analytic topology, Amer. Math. Soc. Colloq. Publ., vol. 28, Amer. Math. Soc., Providence, R. I., 1942.

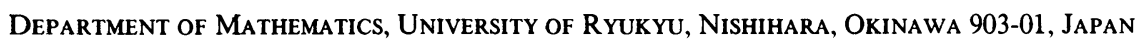

